\title{
Coronary Artery Fistula Draining into Lung Parenchyma Causing Localized Pulmonary Edema: Transcatheter Closure with an Amplatzer Vascular Plug 4
}

\author{
Sanghyok Lim, Se-Whan Lee, Taehun Oh, Donghyun Lee, Minwoo Park, Sujung Han \\ Department of Internal Medicine, Soonchunhyang University Cheonan Hospital, Cheonan, Korea
}

Coronary artery fistula draining into lung parenchymal vasculature has not been reported. Herein, we describe a case of an 81-yearold woman who presented with a localized pulmonary edema on right upper lobe associated with coronary fistula emptied into vasculature in right upper lobe. She underwent transcatheter closure of the fistula with an Amplatzer Vascular Plug 4, which resulted in complete occlusion and improved localized pulmonary edema.

Keywords: Coronary artery fistula; Pulmonary edema; Amplatzer Vascular Plug 4; Vascular closure device

\section{INTRODUCTION}

The incidence of coronary artery fistula is low. Coronary artery fistula usually exists between a coronary artery and a cardiac chamber or another vascular structure [1,2]. Coronary artery fistula draining into lung parenchymal vessel is hard to find. Significant coronary artery fistula has usually hemodynamic change. Because of this, coronary artery fistula considered to be closure [3]. In the past, open heart surgery is treatment of choice, but transcatheter closure technique is a valuable alternative to surgical closure nowadays [3]. We experienced and describe a coronary artery fistula originated from right coronary artery (RCA) and draining to pulmonary vein, into right upper lung field, which was causing localized pulmonary edema at right upper lobe (RUL).

\section{CASE REPORT}

An 81-year-old woman presented to emergency department of Soonchunhyang University Cheonan Hospital with progressive dyspnea. She had a medical history of hypertension and atrial fibrillation. So she had been receiving aspirin, irbesartan, digoxin for ten years at primary clinic. On admission, she was afebrile and chest auscultation revealed crackles at the right upper lung field. Her electrocardiogram showed atrial fibrillation and diffuse non-specific ST change. Echocardiography showed preserved ventricular contractility, moderately enlarged left atrium, and small amount of pericardial effusion. Chest X-ray revealed infiltration at RUL, cardiomegaly, and slight pleural effusion (Fig. 1A). Her troponin-T was normal and brain natriuretic peptide was elevated at 2,181 pg/mL. On hospital day 3, we performed chest computed tomography (CT) and coronary angiography. Chest CT showed patchy consolidation and ground glass opacity in RUL compatible with localized pulmonary edema (Fig. 1B). On coronary angiography, we found a high-flow coronary artery fistula originating from the ostium of RCA and draining into the right upper lung parenchyma (Fig. 2A-C). After treatment with torasemide, spirololactone, low-molecular weight heparin, candesartan, and carvedilol, the patient responded with slightly symptomatic improvement of dyspnea but showed persistent RUL infiltration on chest X-ray. We decided to close the fistula because we thought that hyper-perfusion by the high-flow fistula caused the localized pulmonary edema and impeded clinical improvement. In light of the Amplatzer Vascular Plug (AVP) 4's low profile and its ease of use, we decided to perform transcatheter occlusion by deploying an AVP 4. Informed written consent was ob- 

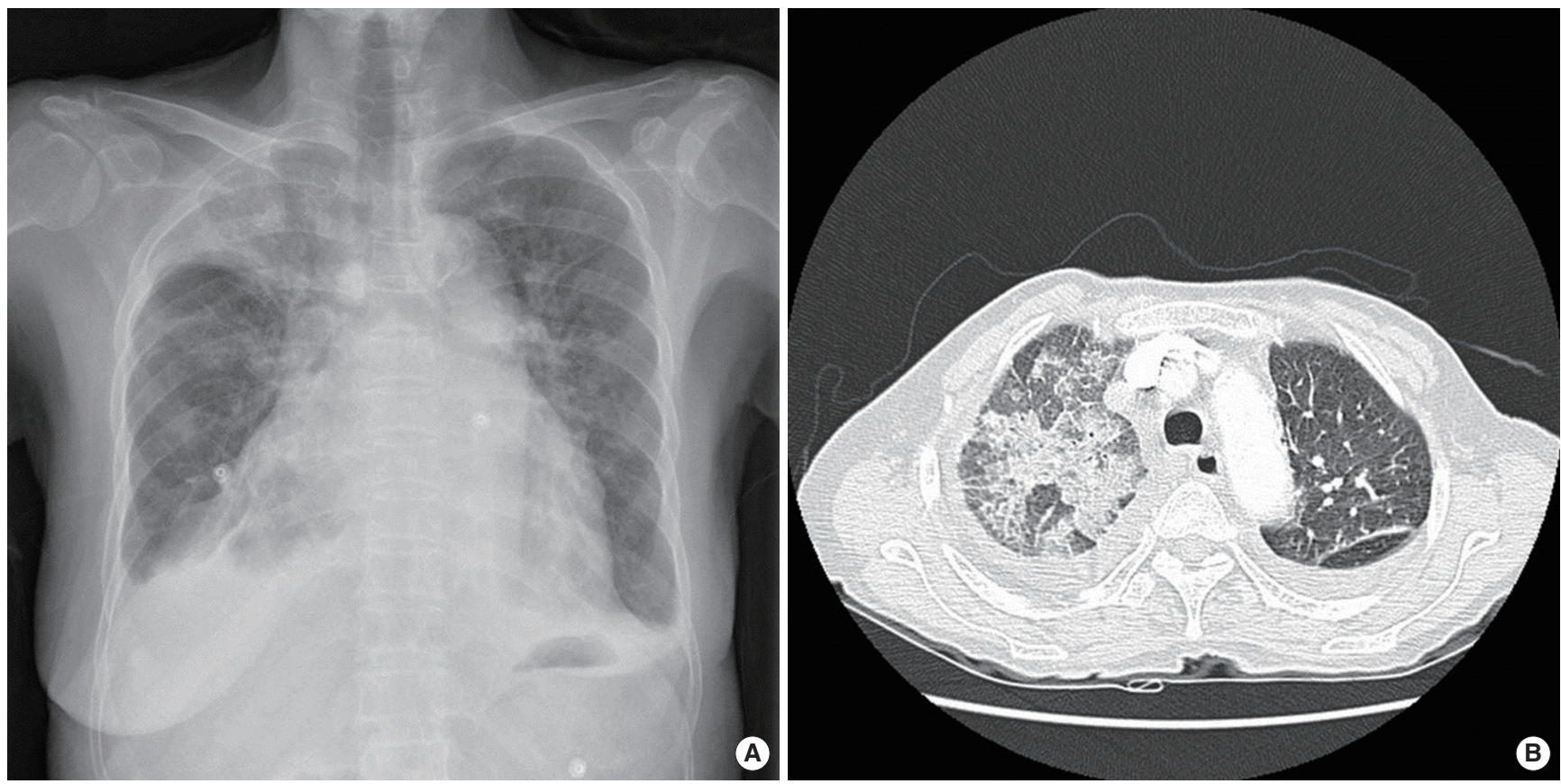

Fig. 1. (A) Initial chest $X$-ray reveals infiltration at right upper lobe (RUL), cardiomegaly, and slight bilateral pleural effusion (A). (B) Chest computed tomography shows patchy consolidation and ground glass opacity in RUL compatible with localized pulmonary edema.
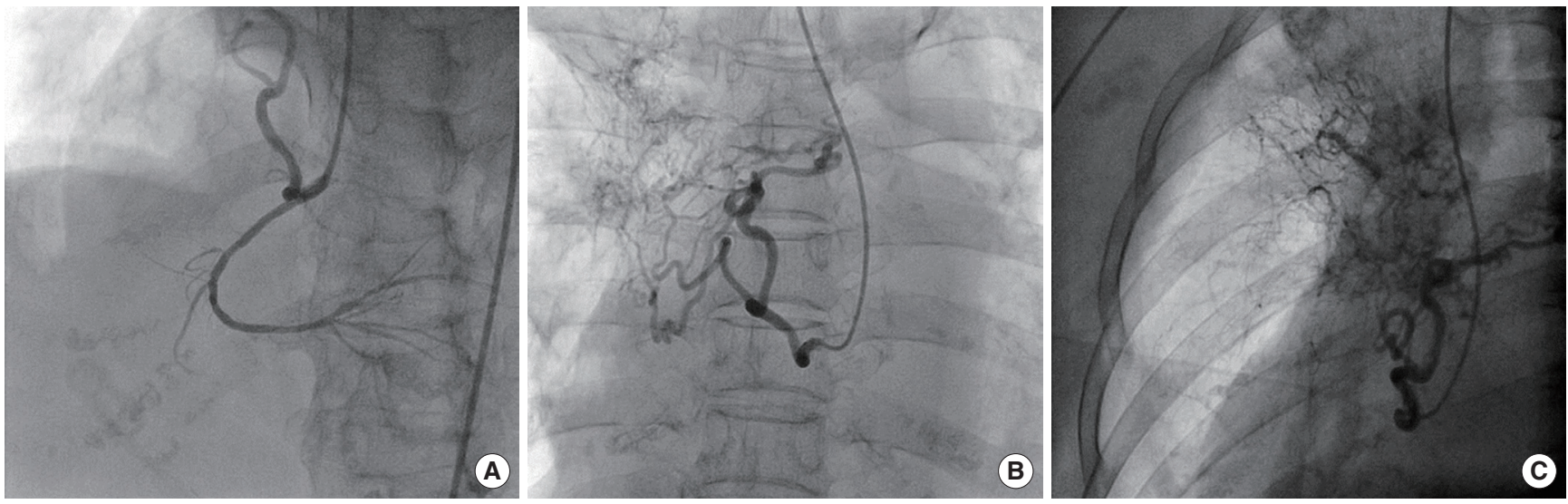

Fig. 2. (A) Selective coronary angiography (left anterior oblique cranial view) shows the coronary fistula originating from the very proximal portion of right coronary artery and (B) draining into the right upper lung field. (C) Subtractive coronary angiography (modified left anterior oblique view) clarifies the draining site of the coronary fistula, directed toward right upper lung parenchyma.

tained, and the patient was readmitted to the cardiac catheterization laboratory on hospital day 7. Vascular access was attained via right femoral artery with 6 French (F) Judkins right (JR) catheter. We passed the $0.36576-\mathrm{mm}$ standard guidewire and performed intravascular ultrasound (IVUS) evaluation for precise assessment about the fistulous tract. At the narrowest segments, which was located at the middle and distal segments of the fistulous tract, vessel diameter was about 4.2 to $4.5 \mathrm{~mm}$ by IVUS measurement. A 5F Heartrail catheter was inserted within a $6 \mathrm{~F}$ JR catheter for better backup support, and was then placed with its tip at the mid-portion of the fistula, a 6-mm AVP 4 was loaded and delivered into the fistula (Fig. 3A). Afterwards, the plug was released from the cable. Although the fistula was not occluded immediately, selective angiography by means of a guiding catheter 5 minutes after plug deployment revealed complete occlusion of the fistula at plug level (Fig. 3B). No periprocedural complications occurred and RUL infiltrate on follow-up chest X-ray dramatically improved (Fig. 4). One week later, the patient was dyspnea-free and in excellent con- 

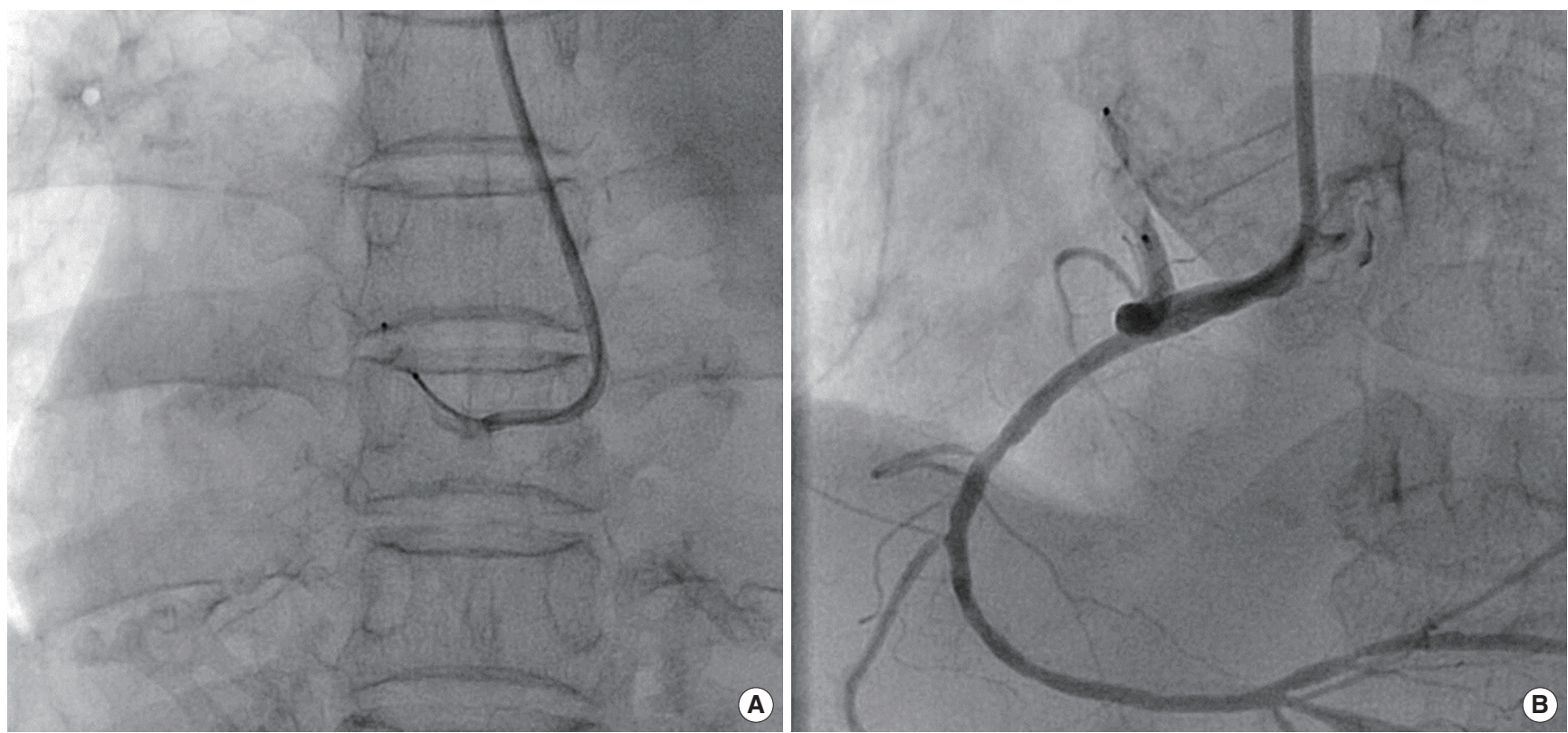

Fig. 3. (A) The Amplatzer Vascular Plug 4 is shown in the middle part of the fistula while it is still connected to the delivery guidewire. (B) Completely occluded fistula, seen at the proximal end of the Amplatzer Vascular Plug 4.

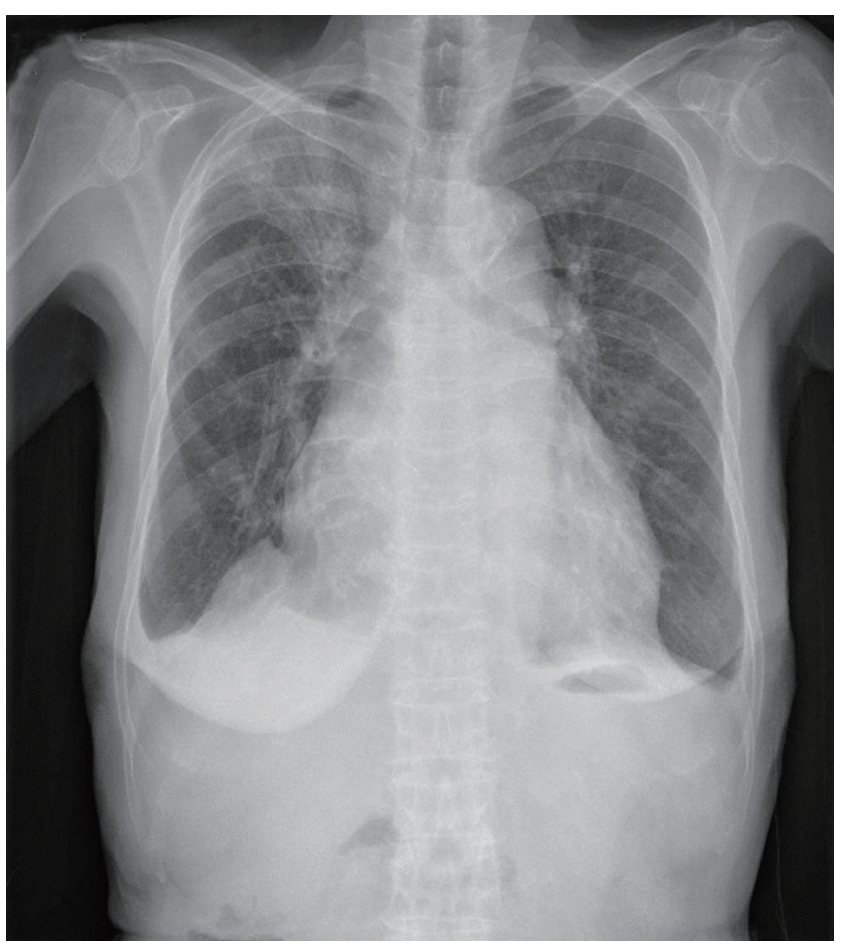

Fig. 4. Post-procedural follow-up chest X-ray showed marked improvement of infiltrate on right upper lobe.

dition and discharged on hospital day 14. During the ensuing 18 months follow-up period, there has been no further episode of heart failure with maintenance of medication.

\section{DISCUSSION}

Coronary artery fistula usually involves a communication between a coronary artery and a cardiac chamber or another vessel $[1,2]$. The clinical features of coronary fistula, which depend on the size and the site of drainage of the fistula, range from asymptomatic manifestation to myocardial ischemia or congestive heart failure $[4,5]$. In medical literature, report of coronary fistula draining into lung parenchymal vasculature is difficult to find. To the best of our knowledge, our case is the first report of coronary fistula draining into lung parenchymal vessel causing localized pulmonary edema at the consistent site of fistula drainage. Localized pulmonary edema has been amply described in the literature in relation to various mechanisms [6,7]. In our case, as a possible mechanism of localized edema, we suggest that regional overflow into RUL due to highflow fistula may cause alveolar flooding, responsible for localized edema formation. The closure of coronary fistula has been recommended in order to prevent complications such as myocardial ischemia, congestive heart failure, arrhythmias, and aneurismal dilation $[5,8]$. The advantages of the transcatheter approach include less morbidity, lower cost, shorter recovery time, and avoidance of thoracotomy and cardiopulmonary bypass [9]. Careful choice of optimal closure device, which depend on various size and location of fistula, is very important for successful transcatheter closure, although given recent advances in transcatheter techniques, most 
coronary fistula can now be closed via transcatheter intervention. In comparison with many other devices that were previously used for the occlusion of coronary fistula, the Amplatzer Vascular Plug affords several advantages, including ease of delivery, a wide range of device sizes, and the opportunity to reposition the device safely during and after initial deployment [10]. In our case, the fistula was relatively small and tortuous. So, we selected the AVP 4 because of its small profile and ease of use for tortuous vessel. Our case is of particular interest, in that the high-flow coronary fistula caused localized pulmonary edema which was firstly reported case and firstly performed transcatheter closure with the AVP 4.

In conclusion, patient with a coronary fistula draining into the lung parenchymal vasculature can cause unusual localized pulmonary edema. In this situation, transcatheter closure or surgical ligation should be considered to prevent aggravation of heart failure. Optimal result of our case suggests that it is feasible and safe to apply an AVP 4 for transcatheter occlusion of relatively small coronary artery fistula. The standardization of this approach, however, will require study of a greater number of cases with longer follow-up periods.

\section{REFERENCES}

1. Roberts WC. Major anomalies of coronary arterial origin seen in adulthood. Am Heart J 1986;111:941-63.

2. Yamanaka O, Hobbs RE. Coronary artery anomalies in 126,595 patients undergoing coronary arteriography. Cathet Cardiovasc Diagn 1990;21: 28-40.

3. Alekyan BG, Podzolkov VP, Cardenas CE. Transcatheter coil embolization of coronary artery fistula. Asian Cardiovasc Thorac Ann 2002;10:4752.

4. Sherwood MC, Rockenmacher S, Colan SD, Geva T. Prognostic significance of clinically silent coronary artery fistulas. Am J Cardiol 1999;83: 407-11.

5. Armsby LR, Keane JF, Sherwood MC, Forbess JM, Perry SB, Lock JE. Management of coronary artery fistulae: patient selection and results of transcatheter closure. J Am Coll Cardiol 2002;39:1026-32.

6. Alarcon JJ, Guembe P, de Miguel E, Gordillo I, Abellas A. Localized right upper lobe edema. Chest 1995;107:274-6.

7. Milne EN, Pistolesi M, Miniati M, Giuntini C. The radiologic distinction of cardiogenic and noncardiogenic edema. AJR Am J Roentgenol 1985;144:879-94.

8. Mavroudis C, Backer CL, Rocchini AP, Muster AJ, Gevitz M. Coronary artery fistulas in infants and children: a surgical review and discussion of coil embolization. Ann Thorac Surg 1997;63:1235-42.

9. Behera SK, Danon S, Levi DS, Moore JW. Transcatheter closure of coronary artery fistulae using the Amplatzer Duct Occluder. Catheter Cardiovasc Interv 2006;68:242-8.

10. Wang W, Li H, Tam MD, Zhou D, Wang DX, Spain J. The amplatzer vascular plug: a review of the device and its clinical applications. Cardiovasc Intervent Radiol 2012;35:725-40. 